\title{
FAUSTINO MIRANDA
}

\section{G. Halffer}

Instituto de Ecología, A.C. Km. 2.5 Carr. Antigua a Coatepec No. 35 i. Congregación El Haya. Xalapa, Veracruz, México.

En una publicación conjunta, el Centro Iberoamericano de la Biodiversidad (CIBIO) de la Universidad de Alicante y el Instituto de Ecología, A.C, de Xalapa, México, rinden homenaje a la vida y obra del botánico hispano-mexicano Faustino Miranda (1905-1964). El libro en cuestión: Mónica Palacios-Ríos y Juvencio A. Carrillo. 2007. Faustino Miranda: Un Botánico Español Apasionado por la Flora Mexicana, CIBIO-Instituto de Ecología, A.C., muy bien ilustrado y documentado, es realmente un homenaje muy afectuoso al que fuera un gran botánico y el iniciador de los estudios modernos de vegetación en México. El interés por Miranda, su vida y su obra, está en pleno auge y en este mismo año acaba de aparecer un segundo libro: Francisco Javier Dosil Mancilla (coordinador). 2007. Faustino Miranda: Una Vida Dedicada a la Botánica, Universidad Michoacana de San Nicolás de Hidalgo, México-Consejo Superior de Investigaciones Científicas, Madrid, también excelente.

No es mi propósito comparar ambos libros que son tanto contribuciones de primer nivel como plenas de información. Si he querido hacer una reflexión sobre lo que para el desarrollo de las Ciencias Biológicas en México representó Miranda, así como de su efecto persistente a través de su obra y continuadores.

Faustino Miranda no fue el único español republicano que contribuyó en forma decisiva al desarrollo de la Biología y en general de la Ciencia en México. Fueron muchos y muy notables. El análisis de su repercusión, más allá de las breves reseñas biográficas ya publicadas, es una tarea pendiente.

En las décadas de 1940 a 1970 la investigación y la enseñanza de la Biología en México, como tantas otras ramas de la ciencia y la cultura, cambiaron radicalmente bajo la influencia de la emigración republicana. El efecto se extiende muchos años más tarde por la labor de los alumnos que formaron. Estos espańoles "trasterrados" que no refugiados, como los llamó el escritor José Bergamín, fueron generosamente acogidos por la sociedad culta mexicana. En unos lugares como la Escuela Nacional de Ciencias Biológicas, integrada el Instituto Politécnico Nacio-

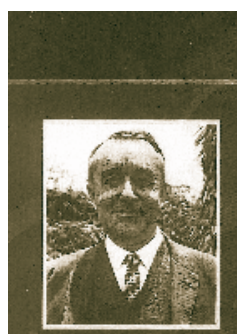

Faustino Miranda un botánico español apasionado por la Flora Mexicana

Mónica Palacios-Rios y Juvencio A. Carrillo $\mathrm{E}$.

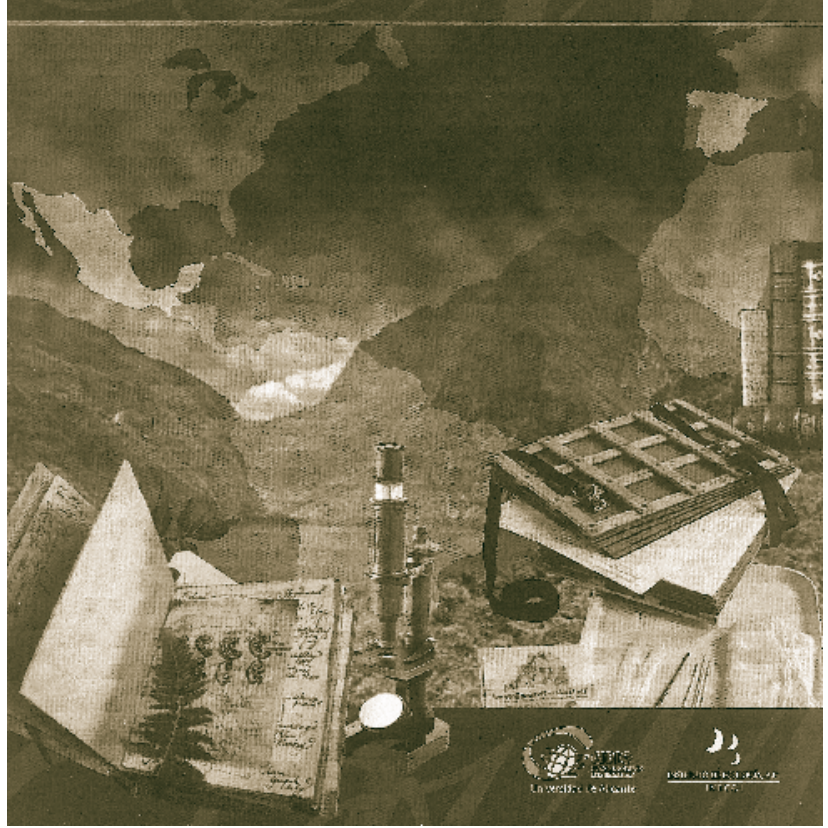


nal creado por el Gral. Lázaro Cárdenas, en forma realmente excepcional. Muchos se nacionalizaron mexicanos y la gran mayoría se incorporaron de tal manera al país, que ni ellos ni los mexicanos establecieron nunca una distinción por su origen. Por mi edad, por ser hijo de un compositor "trasterrado", y por las instituciones en que me formé, conocí a todos ellos (incluyendo a Faustino Miranda) y fui alumno y colaborador de algunos de los más distinguidos (Federico Bonet, Cándido Bolívar y Pieltaín, Manuel Castañeda Aguyo, Dionisio Peláez, etc.). Soy testigo comprometido (uno de los últimos) de los esfuerzos y realizaciones de esa Ciencia Española Trasterrada que acabó siendo tan mexicana.

Faustino Miranda realiza en España, concretamente en Pontevedra, una inicial e importante contribución al conocimiento de las algas (13 trabajos publicados en España, más uno de síntesis publicado en México). A su llegada a México (1939, se naturaliza mexicano en 1941) su atención pasa a las fanerógamas, a las que dedicaría todo el resto de su vida. En 1941 entra al Instituto de Biología de la Universidad Nacional Autónoma de México (UNAM), institución con la que permanecería relacionado hasta su muerte.

Muy importante es su relación con el Estado de Chiapas. Invitado por el Gobernador del Estado es fundador y director del Instituto Botánico de Chiapas de 1949 a 1954. En la capital del Estado crea el primer Jardín Botánico que haría en México, el segundo lo hizo más tarde en la propia UNAM.

Chiapas con su enorme diversidad geográfica y biológica fue el lugar ideal para el desarrollo de sus ideas sobre los tipos de vegetación. La Vegetación de Chiapas en dos volúmenes (primera parte, $334 \mathrm{pp}$, 1952; segunda parte, 426 pp, 1953) es parteaguas en los estudios de vegetación en México. Los cinco años (1949-1954) de estancia en Chiapas, tiempo que pudo dedicar intensamente a recorrer el estado y a su trabajo de investigación, dejaron trece artículos y los dos volúmenes de la Vegetación de Chiapas ya señalados. También un instituto botánico formado con un museo y un jardín botánico.

En los años siguientes Miranda recorrió casi todo el país y continuó publicando sobre los tipos de vegetación que encontraba. En su prolífica carrera dedicó al tema tres libros y veintidós artículos, además de estudios más específicamente taxonómicos (describió setenta taxones entre géneros y especies nuevas) y biogeográficos. Un trabajo de gran repercusión es el hecho en colaboración con Efraín Hernández Xolocotzin: Los Tipos de Vegetación de México y su Clasificación, 1963 (véase bibliografía completa en Palacios-Ríos y Carrillo, 2007). Este trabajo sigue siendo referencia obligada en el estudio de las comunidades vegetales mexicanas. Es interesante señalar los buenos resultados que dio la colaboración Miranda-Hernández: Miranda, ya entonces el mayor conocedor de las fanerógamas de México en su momento y con una amplísima experiencia de campo y el Ing. Efraín Hernández Xolocotzin, especialista en ecología de pastizales y en plantas de interés económico.

Su continuador en el estudio de los tipos de vegetación de México, el gran botánico mexicano Jerzy Rzedowski, dedica su obra clave La Vegetación de México, Editorial Limusa, México, 1978 (hay dos reimpresiones) a Miranda. Debajo de una foto a toda página señala: "incansable forjador de conocimientos botánicos e iniciador de las investigaciones sinecológicas modernas en México”. El mismo Rzedowski dice (op. cit.: 17): "la obra de Miranda no se limita a su prolífica producción, sino también tiene el mérito de haber estimulado el interés por los estudios ecológicos entre sus colegas y estudiantes mexicanos, con lo cual se creó toda una escuela de esta especialidad' (la cursiva es mía).

Como señala Dosil Mancilla (2007) un aspecto de la actividad científica de Miranda que no se ha recalcado suficientemente, es su interés por el manejo de los recursos bióticos. A su regreso a la Ciudad de México (1954) prácticamente participó en todos los ambiciosos proyectos que promovió por aquel entonces el Dr. Enrique Beltrán a través del Instituto Mexicano de Recursos Naturales Renovables y de la propia Subsecretaría Forestal y de la Fauna del Gobierno Mexicano.

Varias e importantes expediciones extendieron su conocimiento a distintas regiones del país (por ejemplo, las zonas áridas, el sureste y el Archipiélago de las Revillagigedo) y ampliaron los horizontes de sus trabajos botánicos. Esta experiencia fue decisiva para poder elaborar los tipos de vegetación de México, país extremadamente variado. 
Entre las instituciones y proyectos con las que estuvo involucrado (además de su investigación y docencia continua en la UNAM) merece especial mención la Comisión de Estudios sobre Ecología de las Dioscóreas Mexicanas de las que Miranda fue activo consejero científico y promotor por cinco años. Esta Comisión (también creación de Enrique Beltrán) vertebró el desarrollo de la botánica mexicana, modernizando la metodología empleada en el estudio de las comunidades vegetales y tuvo un gran papel en la formación y consolidación de jóvenes investigadores, entre ellos figuras tan destacadas como Arturo Gómez-Pompa (que fue su director), José Sarukhán, Javier Valdés, Mario Sousa, Carlos Vázquez Yánez, etc.

A su regreso a México, Miranda se reincorpora a la UNAM (Instituto de Biología y Facultad de Ciencias) y recibe el encargo de crear el jardín botánico de la propia universidad, hasta la fecha el más importante del país, centro de investigación y formación de botánicos y ecólogos vegetales.
Un aspecto que consideramos importante en la producción de Miranda es su participación junto con varios colegas mexicanos en la redacción de los primeros libros de texto de Biología para educación secundaria que tuvimos en México. Estos libros, reimpresos durante varios años, fueron los primeros de gran calidad de que pudimos disponer los estudiantes mexicanos.

Ideas de Miranda y la huella de su personalidad se ha prolongado a través de sus alumnos directos o indirectos. El principal alumno de Miranda ha sido Arturo Gómez-Pompa (véase Gómez-Pompa, A., Recuerdo de mi Relación con Faustino Miranda, en Dosil y Mancilla, 2007). A través de él, tanto en la Comisión de Dioscóreas como en el Jardín Botánico y en general en la UNAM, las ideas de Miranda han tenido y siguen teniendo una influencia directa en la ecología tropical mexicana y en la planificación del uso de los recursos bióticos.

Repetimos, lo ya señalado por Jerzy Rzedowski y que es lo mejor que se puede decir de un investigador: Miranda creó una verdadera escuela. 